\title{
Expression of Her2/neu receptor in carcinoma gallbladder correlates significantly with advanced tumor stage
}

\author{
Farzana Ashai, Aadil Ashraf, Arshad Rashid, Bilal Musharaf Banday, \\ Sunita Bhalla, Shashi Dhawan
}

\begin{abstract}
Aims: Overexpression of Her2/neu is associated with increased disease recurrence and worse prognosis in many cancers. The aim of this study was to correlate Her2/neu positivity with the histopathological features in carcinoma gallbladder. Methods: This study was conducted on 54 cholecystectomy specimens of carcinoma gallbladder in a superspecialty referral center from January 2007 to July 2011. A gross evaluation of tumor, study of histopathology and immunohistochemical staining was done for Her2/neu. The relative frequency of Her2/neu positivity was scored and correlated with other histological prognostic parameters of the tumor. Results: Maximum cases in our study were in stage T2 at the time of diagnosis and most of them were moderately differentiated carcinoma.
\end{abstract}

Farzana Ashai ${ }^{1}$, Aadil Ashraf $^{2}$, Arshad Rashid $^{3}$, Bilal Musharaf Banday ${ }^{2}$, Sunita Bhalla ${ }^{4}$, Shashi Dhawan ${ }^{4}$

Affiliations: ${ }^{1}$ Senior Resident, Department of Pathology, Sheri Kashmir Institute of Medical Science Medical College, Srinagar - 190015; Post Graduate Scholar, Department of Pathology, Sir Ganga Ram Hospital, Rajender Nagar, New Delhi - 110060, India; ${ }^{2}$ Senior Resident, Department of Pathology, Government Medical College, Srinagar - 190010; ${ }^{3}$ Medical Officer, ASYM District Hospital, Budgam, Health Services Kashmir - 190014; ${ }^{4}$ Senior Consultant, Department of Pathology, Sir Ganga Ram Hospital, Rajender Nagar, New Delhi - 110060, India.

Corresponding Author: Dr. Arshad Rashid, G - 22, Shah Anwar Colony, Hyderpora, Srinagar (India) - 190014; Ph: +918494095736; Email: arsh002@gmail.com

Received: 12 June 2015

Accepted: 30 June 2015

Published: 26 September 2015
Her2/neu was strongly positive in five cases (9.25\%) of gallbladder cancer and 14 cases (25.92\%) showed moderate positivity. There was a lot of heterogeneity in the expression of Her2/neu showing $1+$ and $2+$ positivity in different areas. No correlation was seen between immunohistochemical staining pattern and tumor grade, but frequency of Her2/neu positive cases was highest in advanced stage $(p=0.042)$. Conclusion: Her2/neu expression occurs in gallbladder cancer, especially in advanced stage disease and its therapeutic targeting seems promising.

Keywords: Expression, Gallbladder cancer, Her2/ neu, Histopathology, Prognosis

\section{How to cite this article}

Ashai F, Ashraf A, Rashid A, Banday BM, Bhalla S, Dhawan S. Expression of Her2/neu receptor in carcinoma gallbladder correlates significantly with advanced tumor stage. Int $\mathrm{J}$ Hepatobiliary Pancreat Dis 2015;5:86-91.

Article ID: 100040IJHPDFA2015

$* * * * * * * * *$

doi:10.5348/ijhpd-2015-40-CR-15

\section{INTRODUCTION}

Gallbladder cancer (GBC), the most common biliary malignancy, was first described in 1777 by a Viennese 
researcher Maximilian de Stoll [1]. Its incidence parallels the prevalence of gallstone disease; large and longstanding gallstones being associated with a higher risk of GBC [2]. It has a very poor prognosis with only about $32 \%$ five-years survival rate for lesions confined to the gallbladder mucosa that drops to about 10 percent, for more advanced stages. Gallbladder cancer is very common in Chile, Northern India, Japan and Korea [3]. Adenocarcinoma accounts for $80-90 \%$ of cases. There are a number of risk factors that may increase the chances of developing gallbladder cancer that include gallstones and inflammation, polyps, porcelain gallbladder, obesity and Salmonella typhi infection of gallbladder [4]. Her2/ neu (also known as Erb B-2) stands for "Human Epidermal growth factor Receptor 2. Overexpression of this receptor in breast cancer is associated with increased disease recurrence and worse prognosis [5]. Overexpression also occurs in other cancer such as ovarian cancer, stomach cancer, and biologically aggressive forms of uterine cancer, such as uterine serous endometrial carcinoma [68]. There are few but conflicting reports dealing with the clinical significance of expression of Her2/neu in GBC. Moreover, reported immunohistochemical (IHC) over expression ranges from $5-70 \%$ in GBC [9-13]. The aim of this study was to correlate Her2/neu positivity with the histopathological features in carcinoma gallbladder.

\section{MATERIALS AND METHODS}

Material comprised of resected specimens of carcinoma gallbladder examined in the Department of Pathology, Sir Ganga Ram Hospital, New Delhi (India) from January 2007 to July 2011. This study included both retrospective as well as prospective components. The study comprised of 54 cases of GBC found during this period out of 1851 cholecystectomy specimen. A gross evaluation of tumor was done in the resected specimens. After the preliminary study of all Hematoxylin and Eosin stained sections, IHC staining was done for Her2/neu in malignant tumor along with non-tumorous area. The relative frequency of Her2/neu positivity was scored and correlated with other histological prognostic parameters of the tumor. Areas that were not involved by the tumor were also studied for Her2/neu and external positive and negative controls were put while performing IHC staining in each batch. Controls were taken from the known cases of the breast specimen. Her2/neu was graded as per criteria defined by the American Society of Clinical Oncology and the College of American Pathologists [14].

The data thus collected was compiled and analyzed using SPSS version 21 for Mac (IBM Corporation, 2012). Qualitative variables were expressed as proportions in percentages. The association between variables was calculated for 95\% confidence intervals by using "chi square test". A $p$-value < 0.05 was taken as significant. For quantitative data, mean and standard deviation was calculated. An approval for this study was obtained from the Institutional Ethical Committee.

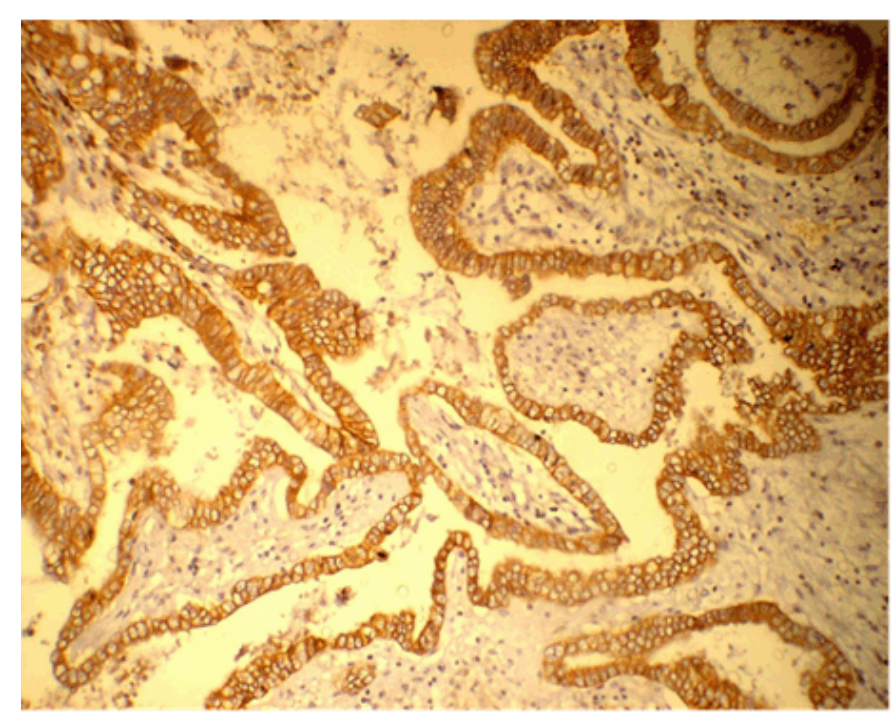

Figure 1: Photomicrograph showing strong and diffuse $3^{+}$ positivity of Her2/neu in Gallbladder Carcinoma (x100).

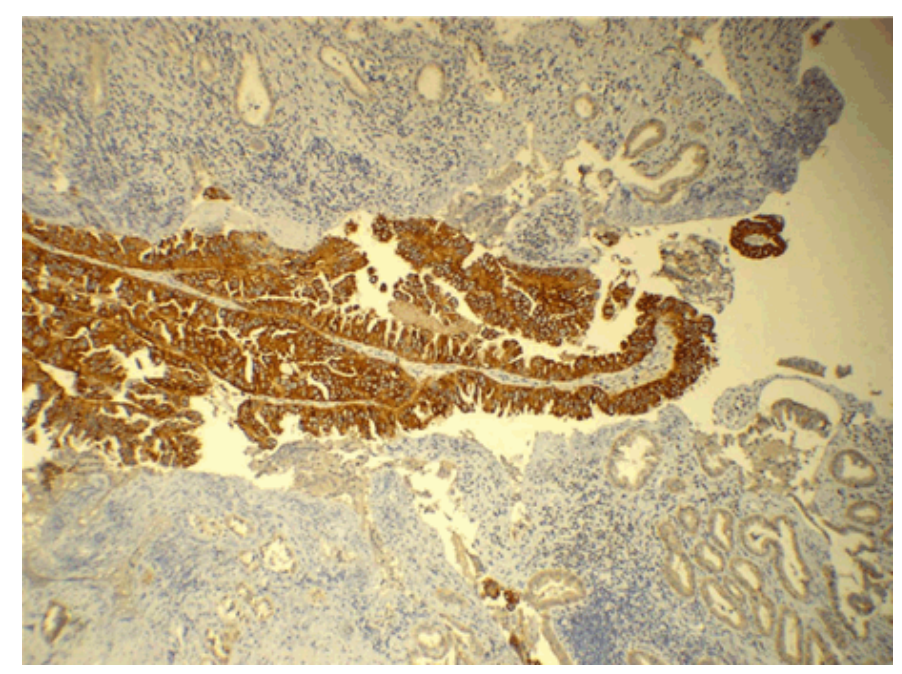

Figure 2: Photomicrograph showing focal $3+$ Her2/neu positivity in a predominant $1+$ area of gallbladder cancer (x100).

\section{RESULTS}

Fifty-four cases in 1851 cholecystectomy specimens were diagnosed as carcinoma of gallbladder (2.9\%). Out of these, 22 cases (1.1\%) were diagnosed after cholecystectomy in routine histopathology without prior clinical diagnosis. Male to female ratio was 1:4.5 and the youngest patient was a 27-year-old female. Twenty-six patients (48\%) had calculi in the gallbladder. Most of the cases (98\%) were adenocarcinoma and only $2 \%$ were adenosquamous carcinoma.

Pyloric metaplasia, intestinal metaplasia and dysplasia were seen in 39 (72\%), 32 (59\%) and 24 cases (44.44\%), respectively. Maximum cases in our study were in stage T2 at the time of diagnosis and most of them were moderately differentiated carcinoma. Carcinomas detected incidentally were mostly in the early stages of cancer (T1). Her2/neu was strongly positive $\left(3^{+}\right)$in 
five cases (9.25\%) and moderately positive (2+) in 14 cases $(25.92 \%)$. There was a lot of heterogeneity in the expression of Her2/neu showing $1+$ and $2+$ positivity in different areas. No correlation was seen between IHC staining pattern and tumor grade (Table 1), but frequency of Her2/neu positive cases was highest in advanced stage $(p=0.042)$ (Table 2).

\section{DISCUSSION}

Gallbladder cancer is the most common cancer of the biliary tract and has a particularly high incidence in Chile, Japan, and Northern India. Its incidence steadily increases with age and women are affected two to six times more often than men [3]. High incidence of GBC in our region and its poor prognosis necessitates a closer look at the molecular changes for evolving an effective early diagnostic and therapeutic strategy. Expression of HER2/neu has been intensively studied in different tumor entities and has led to the use of targeted therapy with specific inhibitors or antibodies of these receptors in colorectal, breast, lung as well as head and neck cancer. Its expression as potential therapeutic targets has been reported in various tumors [6-8]. For biliary tract carcinoma, data for HER2/neu overexpression have been presented in mostly small patient cohorts. Its overexpression and amplification has been found in a range between $5 \%$ and $76 \%$ in biliary tract cancers [913]. Targeted therapy with the anti-HER2/neu antibody in breast cancer is effective when the HER2/neu receptor is overexpressed [5]. However, its study in gallbladder carcinoma is limited.

In the present study, histopathological examination of 54 gallbladder carcinoma cases was done in our institute and it was correlated with Her2/neu IHC staining. The youngest patient in our study was 27 years and the oldest was 86 years. To the best of our knowledge, the youngest patient of carcinoma gallbladder reported in literature is a 15-year-old girl reported by Khandelwal et al. in their study of 150 cases of GBC [15]. The mean age of presentation in our series was 57.31 years. The maximum numbers of patients were in the seventh decade $(33.33 \%)$ followed by fifth decade (29.3\%).

The association between cholelithiasis and gallbladder cancer has been known since 1861 . However, it is seen that only $0.5^{-1} \%$ of cases with gallstone disease develop carcinoma of gallbladder $[16,17]$. In the study by Mohan et al. $1 \%$ of cholecystectomies performed for cholelithiasis showed early carcinoma [18]. We found $2.9 \%$ of gallbladder cancer out of a total of 1851 cholecystectomies, out of which 22 (1.1\%) were incidental carcinoma. In our study, 26 patients ( $48 \%$ ) of gallbladder cancer had calculi in the gallbladder.

Fifty-three of our cases (98\%) were adenocarcinoma and one case (2\%) was adenosquamous carcinoma.

Table 1: Correlation of immunohistochemical staining with tumor differentiation

\begin{tabular}{llllll} 
Differentiation & Negative & $1+$ & $2+$ & $3+$ & Notal \\
Well differentiated & 1 & $\begin{array}{l}9 \text { (one with focal } 3+ \\
\text { positivity) }\end{array}$ & 1 & $4(14.81 \%)$ & 27 \\
Moderately differentiated & 7 & 4 & 12 & 1 (with focal $3+$ & $1(6.25 \%)$ \\
$\begin{array}{l}\text { Poorly } \\
\text { Differentiated }\end{array}$ & 2 & 12 & positivity) & 16 \\
Total & $10(18.51 \%)$ & $25(46.29 \%)$ & $14(25.92 \%)$ & $5(9.25 \%)$ & 54 \\
\hline
\end{tabular}

Table 2: Correlation of immunohistochemical staining with staging $(p<0.05)$

\begin{tabular}{|c|c|c|c|c|c|}
\hline Staging & Negative & $1+$ & $2+$ & $\mathbf{3}^{+}$ & Total \\
\hline $\mathrm{T} 1$ & 1 & $\begin{array}{l}9 \text { ( } 1 \text { with focal } 3^{+} \\
\text {positivity) }\end{array}$ & 1 & Zero & 11 \\
\hline T2 & 7 & 13 & $\begin{array}{l}13 \text { (one with focal } \\
3+\text { positivity) }\end{array}$ & $3(8.33 \%)$ & 36 \\
\hline $\mathrm{T} 3$ & 2 & 3 & Zero & $2(28.57 \%)$ & 7 \\
\hline Total & 10 & 25 & 14 & $5(9.25 \%)$ & 54 \\
\hline
\end{tabular}


Levin et al. [19] and Rajagopalan et al. [20] reported over $85-90 \%$ of gallbladder carcinoma histologically as adenocarcinoma in their studies. Twenty-two cases (41\%) in our study were diagnosed incidentally. Out of which seven cases (32\%) were in stage $\mathrm{T} 1$, twelve cases (55\%) in stage T2 and three cases (13\%) were in stage T3. On the other hand, out of 32 previously diagnosed cases of gallbladder carcinoma, 24 cases $(75 \%)$ were in stage T2 and four cases (12.5\%) each in stage $\mathrm{T} 1$ and T3. It reflects the fact that most of the cases diagnosed incidentally are in the early stages of cancer (T1). Shih et al. reported incidentally discovered GBC during cholecystectomy in 53 patients out of 107 patients of GBC (50\%) at Johns Hopkins Hospital in a 10-year study period [21]. They concluded that patients who were found to have gallbladder carcinoma incidentally during cholecystectomy had a significant increase in survival when compared with those who were admitted electively with a known diagnosis. In our study, 36 (66.66\%) of the cases were in stage $\mathrm{T} 2$; eleven (20.37\%) in stage $\mathrm{T} 1$, and seven (13\%) in stage $\mathrm{T}_{3}$. Memon et al. reported $61 \%$ gallbladder cancers in clinical stage IV, $22 \%$ in stage III and $17 \%$ in stage II at the time of presentation [22].

Her2/neu was strongly positive in five cases (9.25\%) of gallbladder cancer in our study, out of which four were moderately differentiated adenocarcinoma and one was poorly differentiated adenocarcinoma. This finding was in accordance with the findings of Puhalla et al. who found expression of HER2/neu in $13 \%$ of the patients of GBC with IHC staining [23]. Similar IHC pattern was seen between the tumor cells and dysplastic cells. Adjacent normal mucosa was negative for Her2/neu staining in all except one case in our study. Kawamoto et al. reported positive overexpression of Her2/neu in GBC in $31.2 \%$ of specimens by IHC method [9].

Only few studies of overexpression of Her2/neu in gallbladder cancer have been reported from India. Chaube et al. investigated the role of Her2/neu in the carcinogenesis of gallbladder by IHC and found $25 \%$ of cases positive for Her2/neu expression [24]. In their study, positivity decreased with increasing grade of gallbladder carcinoma. They have concluded that decreasing over expression of c-erbB-2 with increasing grade suggest that c-erbB-2 is likely to play a lesser role in tumor progression. In our study we did not find any such correlation. However, there seems to be some correlation of IHC staining pattern and tumor stage. There were 11 cases in stage $\mathrm{T} 1,36$ cases in stage $\mathrm{T} 2$ and 7 cases in $\mathrm{T} 3$ stage. Out of these case $3+$ positivity was shown by 2 cases $(28.57 \%)$ in stage $\mathrm{T}_{3}$; three cases (8.33\%) in stage T2 and none in stage T1. Hence the frequency of Her2/ neu positive cases was highest in advanced stage ( $p=$ o.042). Study with larger number of cases is required to further substantiate this finding.

In our study, one case each of well differentiated and a poorly differentiated tumor, showed sharply defined $3^{+}$ positivity adjacent to $1+$ positive area. Also there were few cases in which focal $2+$ positive areas were present adjacent to predominant $1+$ positive area. This suggests that even in a single case of GBC, different staining patterns of Her2/neu can be seen which highlights the importance of adequate sampling of the tumor for Her2/ neu immunostaining. To the best of our knowledge, such a heterogeneous pattern of immunostaining of Her2/neu has never been reported in literature prior to this study.

Also equivocal IHC staining (2+) cases are an important part of the ongoing discussion. Next to IHC staining to evaluate Her2/neu protein overexpression, a second line gene amplification test is generally deemed necessary for these cases [25]. In a study done by Kawamoto et al. $26.7 \%$ of equivocal immunohistochemistry were fluorescent in situ hybridization (FISH) positive in GBC [9]. Hence, if we had done the gene amplification in equivocal cases $(2+)$ of GBC, which included 14 cases (25.92\%) in our study, the percentage of Her2/neu positivity would have gone still higher; however, it was not done as it was outside the protocol of the study.

\section{CONCLUSION}

In view of aggressive nature of gallbladder carcinoma and lack of good therapeutics, there is an urgent need for identification of cancer specific cellular targets that form the basis of translational therapeutics in future. One of the important conclusions of our study is that as many cases of gallbladder cancer express Her2/neu, its therapeutic targeting seems to be promising.

$$
* * * * * * * * *
$$

\section{Author Contributions}

Farzana Ashai - Substantial contributions to conception and design, Acquisition of data, Analysis and interpretation of data, Drafting the article, Revising it critically for important intellectual content, Final approval of the version to be published

Aadil Ashraf - Analysis and interpretation of data, Drafting the article, Revising it critically for important intellectual content, Final approval of the version to be published

Arshad Rashid - Analysis and interpretation of data, Drafting the article, Revising it critically for important intellectual content, Final approval of the version to be published

Bilal Musharaf Banday - Analysis and interpretation of data, Drafting the article, Revising it critically for important intellectual content, Final approval of the version to be published

Sunita Bhalla - Substantial contributions to conception and design, Acquisition of data, Analysis and interpretation of data, Drafting the article, Revising it critically for important intellectual content, Final approval of the version to be published 
Shashi Dhawan - Substantial contributions to conception and design, Acquisition of data, Analysis and interpretation of data, Drafting the article, Revising it critically for important intellectual content, Final approval of the version to be published

\section{Guarantor}

The corresponding author is the guarantor of submission.

\section{Conflict of Interest}

Authors declare no conflict of interest.

\section{Copyright}

(C) 2015 Farzana Ashai et al. This article is distributed under the terms of Creative Commons Attribution License which permits unrestricted use, distribution and reproduction in any medium provided the original author(s) and original publisher are properly credited. Please see the copyright policy on the journal website for more information.

\section{REFERENCES}

1. Diehl AK. Epidemiology of gallbladder cancer: a synthesis of recent data. J Natl Cancer Inst 1980 Dec;65(6):1209-14.

2. Vitetta L, Sali A, Little P, Mrazek L. Gallstones and gall bladder carcinoma. Aust N Z J Surg 2000 Sep;70(9):667-73.

3. Lai $\mathrm{CH}$, Lau WY. Gallbladder cancer-a comprehensive review. Surgeon 2008 Apr;6(2):101-10.

4. Mittal R, Jesudason MR, Nayak S. Selective histopathology in cholecystectomy for gallstone disease. Indian J Gastroenterol 2010 Sep;29(5):211.

5. Slamon DJ, Clark GM, Wong SG, Levin WJ, Ullrich A, McGuire WL. Human breast cancer: correlation of relapse and survival with amplification of the HER-2/neu oncogene. Science 1987 Jan 9;235(4785):177-82.

6. Schuell B, Gruenberger T, Scheithauer W, Zielinski Ch, Wrba F. HER 2/neu protein expression in colorectal cancer. BMC Cancer 2006 May 8;6:123.

7. Mitra AB, Murty VV, Pratap M, Sodhani P, Chaganti RS. ERBB2 (HER2/neu) oncogene is frequently amplified in squamous cell carcinoma of the uterine cervix. Cancer Res 1994 Feb 1;54(3):637-9.

8. Beckhardt RN, Kiyokawa N, Xi L, et al. HER-2/ neu oncogene characterization in head and neck squamous cell carcinoma. Arch Otolaryngol Head Neck Surg. 1995 Nov;121(11):1265-70.

9. Kawamoto T, Krishnamurthy S, Tarco E, et al. HER Receptor Family: Novel Candidate for Targeted Therapy for Gallbladder and
Extrahepatic Bile Duct Cancer. Gastrointest Cancer Res. 2007 Nov;1(6):221-7.

10. Roa I, de Toro G, Schalper K, de Aretxabala X, Churi C, Javle M. Overexpression of the HER2/ neu Gene: A New Therapeutic Possibility for Patients With Advanced Gallbladder Cancer. Gastrointest Cancer Res 2014 Mar;7(2):42-8.

11. Suzuki T, Takano Y, Kakita A, Okudaira M. An immunohistochemical and molecular biological study of c-erbB-2 amplification and prognostic relevance in gallbladder cancer. Pathol Res Pract 1993 Apr;189(3):283-92.

12. Kamel D, Pääkkö P, Nuorva $K$, Vähäkangas $K$, Soini Y. p53 and c-erbB-2 protein expression in adenocarcinomas and epithelial dysplasias of the gall bladder. J Pathol 1993 May;170(1):6772.

13. Kim YW, Huh SH, Park YK, Yoon TY, Lee SM, Hong SH. Expression of the c-erb-B2 and p53 protein in gallbladder carcinomas. Oncol Rep 2001 Sep-Oct;8(5):1127-32.

14. Allison KH, Dintzis SM, Schmidt RA. Frequency of HER2 heterogeneity by fluorescence in situ hybridization according to CAP expert panel recommendations: time for a new look at how to report heterogeneity. Am J Clin Pathol 2011 Dec;136(6):864-71.

15. Khandelwal M, Khandelwal C. Surgery for advanced gall bladder cancer. In: Taylor I, Johnson CD, editors. Recent Advances in Surgery 34. 1st ed. Vol. 34. New Delhi: Replika Press Pvt. Ltd. Jaypee Brothers; 2011. pp. 57-71.

16. DiehlAK. Gallstonesizeand the risk of gallbladder cancer. JAMA 1983 Nov 4;250(17):2323-6.

17. Lowenfels AB, Walker AM, Althaus DP, Townsend G, Domellöf L. Gallstone growth, size, and risk of gallbladder cancer: an interracial study. Int J Epidemiol 1989 Mar;18(1):50-4.

18. Mohan H, Punia RP, Dhawan SB, Ahal S, Sekhon MS. Morphological spectrum of gallstone disease in 1100 cholecystectomies in North India. Indian J Surg 2005;67:140-2.

19. Levin B. Gallbladder carcinoma. Ann Oncol 1999;10 Suppl 4:129-30.

20. Rajagopalan V, Daines WP, Grossbard ML, Kozuch P. Gallbladder and biliary tract carcinoma: A comprehensive update, Part 1. Oncology (Williston Park) 2004 Jun;18(7):88996.

21. Shih SP, Schulick RD, Cameron JL, et al. Gallbladder cancer: the role of laparoscopy and radical resection. Ann Surg 2007 Jun;245(6):893-901.

22. Memon MA, Anwar S, Shiwani MH, Memon B. Gallbladder carcinoma: a retrospective analysis of twenty-two years experience of a single teaching hospital. Int Semin Surg Oncol 2005 Mar 17;2(1):6. 
23. Puhalla H, Wrba F, Kandioler D, et al. Expression of p21(Wafl/Cip1), p57(Kip2) and HER2/neu in patients with gallbladder cancer. Anticancer Res 2007 May-Jun;27(3B):1679-84.

24. Chaube A, Tewari M, Garbyal RS, Singh U, Shukla HS. Preliminary study of p53 and c-erbB-2 expression in gallbladder cancer in Indian patients manuscript id: 8962091628764582. BMC Cancer 2006 May 10;6:126.
25. Koutras A, Kalogeras KT, Wirtz RM, et al. Evaluation of the prognostic significance of HER family mRNA expression in high-risk early breast cancer: a Hellenic Cooperative Oncology Group (HeCOG) validation study. J Transl Med 2015 May 29;13:171.
Access full text article on other devices

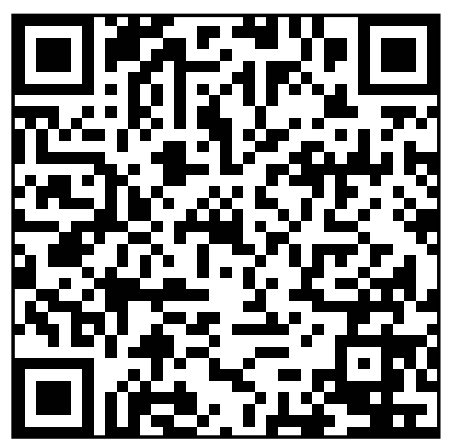

Access PDF of article on other devices

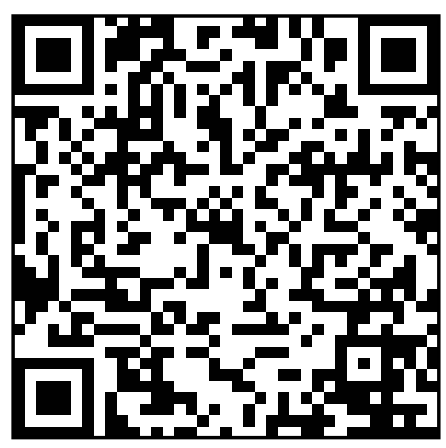

